\title{
Gendering disability and disabling gender: Critical reflections on intersections of gender and disability
}

Kharnita Mohamed and Tamara Shefer

\section{Introduction}

Discourses of normalcy are deeply imbricated in the construction of the social world and organise relations between persons, persons and the State, persons and institutions and intrapsychic relations. Conversely, discourses about pathology and the abnormal underpin the regulation and disciplining of subjectivities intersected with ideas about race, gender, sexuality, class, ethnicities, nationalisms, and other identity vectors.

The normal/abnormal binary is profoundly interwoven into existing power and privilege. The construction of normalcy rationalises and bolsters the marginalisation and 'othering' of bodies, minds, affects, and sexualities that do not fit into a particular culture's imaginary of the ordinary, everyday or acceptable. The suppression of diversity, the negation of potential futures (and presents and pasts) and notions about the im/perfectibility of the human are infused with fears about disability. Disability discourse is thus pervasive, is invariably normalised and ranges through ideas of ideal bodies, ideal relations, ideal psyches, ideal institutions, and nation States.

For example, disability discourse provides the impetus and biopolitical logic for neoeugenics projects centred on genome testing, and undergirds ideas about parenting and reproductive choice and what kinds of people count as fully human. The relations between reproduction and disability are mediated through women's bodies, which are often the battlegrounds through which normalcy is negotiated. Regardless of whether a decision is pro-life or pro-choice, multiple discourses converge and are mobilised to make a seemingly normative choice about termination when a foetus presents as 'risky' or 'defective' or 'disabled': medical, legal, social, bioethical, cultural, religious, gendered notions of parenting and care, ideas about the foetus/child's possibilities for labour and work, the foetus/child's possibility for interacting as a social being and for loving and reproducing in the future. In other words, all the congealed discourses that determine who counts as human.

It is the ideology of ability (Siebers, 2008) or the discourse of normalcy (Davis, 2006) that provides the horizon of possibilities or limitations through which these bioethical considerations are made. This is merely one of myriad examples in which 
disability prefigures, modifies and codifies possibilities and regulates everyday life. Disability discourse therefore affects everyone.

As an embodied possibility, 97\% of impairments are acquired rather than congenital and about $10 \%$ of the population has a disability (Goodley, 2011). Accidents, ageing, warfare, food insecurity, poverty, structural violence, gendered violence and its varied manifestations, unsafe labour practices, limited healthcare services and all the ongoing vicissitudes of living in fragile bodies with sensitive psyches means impairment in some form or other awaits most people. Breaking the binary of disabled and able-bodied, the term Temporarily Able Bodied (Marks, 1999) is often used by disability activists to extend solidarity.

Disability studies provides crucial insights into, amongst others, subjectivity, identities, embodiment, epistemology, ontology, methodology, pedagogy, practices and resources for citizenship, representation, the relation between language and materiality, institutional praxis, exclusion and inclusion, capital, liberalism and neoliberalism, spatiality and the built environment. Disability figures in racialised and heteronormative ideas about sexualities when certain sexualities like LGBT and black desire and sexual expression are pathologised and treated as deviant. Un/desirable body types, female genital cutting and male circumcision, whether bodies should have either a penis or a vagina and be corrected if they have both, ideas about women's hysteria and black men's hypermasculinity, are mediated by discourses on 'proper' gendered behaviour and bodies.

Normative notions of ability/disability may also shape whether buildings need stairs or ramps and how the built environment is configured. Communicative practices and pedagogy are informed by dis/ability discourse such as whether writing should be visual or tactile, language should be spoken or signed, what kinds of learning count at schools and what forms of intelligence are socially valuable and marketable.

This is by no means a comprehensive catalogue. As a category of analysis, disability enables a range of entry points into what it means to be human and what the conditions for inclusion and exclusion are.

Disability is deeply gendered (Fine and Asch, 1988; Gerschick, 2000; GarlandThomson, 2002; Smith and Hutchison, 2004; Wendell, 2006; Hall, 2011). Women are invariably adversely affected by disability (Fine and Asch; 1988; Smith and Hutchison, 2004; Hall, 2011). Disabled women tend to be undereducated and poorer than their male counterparts (Emmett, 2006), more likely to be at risk from sexual abuse, are forcibly sterilised and tend to be desexualised (Sait et al, 2009). Disabled men develop strategies in relation to hegemonic masculinity (Gerschick and Miller, 200o) and destabilise the conjunctures of masculinity and patriarchal privilege (Shuttleworth et al, 2012). 
Globally disability studies is a flourishing field of study with a burgeoning literature. Following the global politics of knowledge production, most disability theorisation has emerged from Euro-America and Australia. Although there has been wonderful pioneering work done on disability in South Africa (e.g. Watermeyer et al, 2006; MacLachlan and Swartz, 2009; Sait et al, 2009; Popplestone, 2009) with an emergent literature, disability is still understudied in Southern Africa and the rest of the continent.

There are very few studies of disability from a feminist or gendered perspective. This special edition attempted to address this lacuna and brings together papers that worked through the intersection of disability and gender. Most of the papers focus on women's experience of disability, and very few disarticulate types of disability. Articles included here foreground the intersectionality of disability for women which shapes disabled women's experiences of being in the world, characterised by practices of stigma, marginalisation and 'othering'.

Prominent themes represented in the issue include: experiences of sexuality and relationship, motherhood, gender-based violence (GBV), challenges in leadership, representations of gendered disability in popular and public contexts, and resistance and challenge to dominant negative constructions of disabled women.

We provide a brief overview of some of the most important models of disability in the first section; the second section sketches the intersection of gender and disability; the third feminist disability studies; and the fourth some of the work that has emerged on disability in Africa. The final section introduces the papers.

\section{Defining disability}

Disability is varyingly defined and definitions change across time and space. As with most definitions, disability is wrought through epistemological imaginaries which affect what counts as disability, which subjects are subsumed under the label and the effects that are manifested and rendered visible in their lives. Disability tends to be separated into physical impairments, mental and learning impairments, sensory impairments, and emotional and psychological impairments.

\section{Models of disability}

Dan Goodley (2011: 1-21) provides an overview of some of the most prominent models of disability: medical, social, cultural, the Nordic relational model, a postcolonial model and the World Health Organization (WHO)'s biopsychosocial 
model. Viewing disability as a personal tragedy in need of cure and rehabilitation invariably draws on the medical model of disability. To explain disability as an act of God is to invoke a moral position on disability. Both these discourses disability as pathology and disability as evidence of sin in need of divine intervention - locate disability in the individual. Individualising discourses of disability reduces people with disabilities' agency, is essentialist, attempts to fix people with disabilities rather than disabling contexts, expands the power of health and social care professionals and reinforces the discourse of normalcy.

Emerging out of political activism in the United Kingdom for disabled people in the 1980 s, the social model of disability made a distinction between impairment and disability. Impairment was the physiological limitation, whilst disability was the disabling social and material conditions experienced by disabled people. In the United States of America a minority group model, inspired by the civil rights movement and queer politics, challenged the hegemony of ableism. People-first language was encouraged, to recognise the person before the disability. Both models disrupted individualising discourses of disability to scrutinise the relationship between impairment and disability. In so doing, attention was brought to the contexts within which disabled people are marginalised, excluded and stigmatised, as well as the positive impacts of disability communities.

The engagement by predominantly North American humanities scholars with disability has led to a cultural model of disability which explores the work that disability does as a cultural construction to regulate 'normalcy'. They do not separate disability from impairment as they argue that biology and culture are entangled and are not mutually exclusive. The Nordic relational model of disability focuses on how disability is created through the relational processes of: (1) the mis/match of the person/environment; (2) the context; and (3) disability as a relative construct. Disability is also defined through other foci such as economic models, human rights, and customer service.

Disability theorisation is overwhelmingly Northern, even though concepts may not always translate easily into other contexts. Livingston $(2005,2006)$ shows how the orthodoxies of contemporary disability studies' ideas of social exclusion and normalcy, for example, differ in Botswana. Unlike in the North, independent living is not highly valued in Botswana, as people understand themselves as interdependent. Whereas for Northern scholars social relationships produce disability, Batswana believe impaired relationships produce misfortune which becomes disability. Further, the politics of knowledge silences the complicity of the global North in producing impairment in the South (see Meekosha, 2011). Meekosha (2011) asserts the importance of postcolonial settler colonies theorising disability from their own perspectives, as often the relationship with the global North produces disabling conditions and impairment for people in the South. Priestley (2006) demonstrates the challenge of rethinking disability from a 
Southern perspective. Whilst he argues for a distinctly Southern African disability studies programme which reflects local need and understandings, he borrows heavily from a Northern conceptual apparatus which reproduces Northern concepts of personhood and organisation of social relations.

To bridge the divide of the medical and social models, the WHO has devised the International Classification of Functioning, Disability and Health (ICFDH), which draws upon what they call a biopsychosocial model of disability (WHO, 2011). The ICFDH acknowledges that disability is a complex phenomenon and centres around functional limitations of the body, participation and activities. The supranational definition has been critiqued for the focus on functional limitation (see Siyabulela and Duncan, 2006), standardisation and the flattening of regional difference, which corresponds to global relations of power (Goodley, 2011) and that disability as a category of social classification does not necessarily travel across cultures (Ingstad and Whyte, 1995; Ginsburg and Rapp, 2013).

Nonetheless, the ICFDH was intended to provide comparable data and was used to measure disability prevalence for the 2011 WHO World Report on Disability. An estimated 650 million (15.6\%) people in 2004 over the age of 18 experienced significant difficulty in functioning. In lower-income countries the average disability prevalence was $18 \%$; it started at $11.8 \%$ in high-income countries. For people over 60 in lower-income countries the prevalence was $43.4 \%$, compared to $29.5 \%$ in higher-income countries. Globally, vulnerable groups like women, the poor and older people had higher rates of disability prevalence.

\section{Gender and disability}

Data that focus on gendered experiences of disability are sparse (Fine and Asch, 1988), and given what we do know, building a reliable knowledge base is urgent. Literature started emerging on women's experiences of disability in the 1980 os (e.g. Asch and Fine, 1988; Browne et al, 1985; Deegan and Brooks 1985).

Many studies of disability tend to treat people with disabilities as a monolithic and ahistorical group. The type of disability, its severity and visibility combined with age of onset will determine the degree of gendered expectations, and thus gendering is conditional for disabled people (Gerschick, 2000). Nonetheless, to provide a sense of the scope of the gendered dynamics, according to the WHO (2011) female disability prevalence is $19.2 \%$ while it is $12.0 \%$ for men; ${ }^{1} 50.6 \%$ of boys with disabilities have completed primary school and $41.7 \%$ girls. In lowincome countries $20.1 \%$ of disabled women are likely to be employed in comparison with men at $58.6 \%$. 
Asch and Fine (1988) famously stated that women with disabilities face sexism without the pedestal. Disabled women are infantilised and imagined as helpless victims (Asch and Fine, 1988; Barnes and Mercer, 2010). Despite their greater risk for sexual violence, they are treated as asexual and are expected to forego motherhood as they are seen as transmitters of faulty genes or as incapable of raising a child. Groce et al (2009) found that women with disabilities were three times more likely to experience emotional, sexual and physical abuse than non-disabled women. Women with disabilities have lower survival rates than men with disabilities, more unstable relationships, and thus less social protection from abuse.

Women with disabilities experience higher maternal morbidity and mortality rates due to lack of reproductive and sexual health education, and are often provided with less health care because they are expected to be asexual. Women with disabilities experience more poverty (Emmet, 2006) and social isolation, and have less support and access to services (Abu Habib, 1995, 1997). Women are also less likely to receive timely healthcare interventions, which may lead to disabling conditions (see Abu Habib, 1997) and therefore more older women than men have disabilities (WHO, 2011). Mothers of disabled children are subjected to blaming discourses, are twice as likely to be deserted, face social isolation and are expected to be the primary caregiver, financially and emotionally (Abu Habib, 1995; Groce et al, 2009).

Although small, there is a growing body of work on masculinity and disability. The stigma of disability is in tension with masculine privilege and renders the patriarchal dividend unstable (Gerschick, 2000), which Shuttleworth et al (2012) call the dilemma of disabled masculinity. Men with disabilities tend to identify with hegemonic ideals of masculinity such as physical strength, independence and bravado, which places them in conflict with their marginalised status (Hahn, 1989). Gerschick and Miller (2000) found that men responded to the conflict with their masculinity by either relying on hegemonic masculine ideals, reformulating them or rejecting them. The men in Gerschick and Miller's (2000) study did not completely fit into one of the three categories, and the boundaries between these responses are not clear-cut (see also Gerschick, 1998). The responses to disability thus differ amongst men but also within men (see also Shakespeare, 1999, 2006).

\section{Feminist disability studies}

Disabled women were largely rendered invisible within the feminist movement until fairly recently (Asch and Fine, 1988; Garland-Thomson, 2002; Hall, 2011). Feminist imaginaries of strong, powerful competent women were in conflict with stereotypes of disability (Asch and Fine, 1988). Rosemarie Garland-Thomson (2011) says her first usage of the term 'feminist disability 
studies' was intended to fuse feminist theory with disability studies and materialise it as an extant critical field.

In her influential essay 'Integrating disability, transforming feminist theory', Garland-Thomson (2002:1) stated that "integrating disability as a category of analysis and a system of representation deepens, expands, and challenges feminist theory". Interpenetrating disability studies and feminist theory enables complex analyses of embodiment, representation, identity and activism. Thinking through disability dislodges normative ideas of what it means to be human and disrupts the ability/disability system which marks and differentiates bodies.

To contend with disability as a ubiquitous and regulatory discourse is to no longer flee from the 'rejected body' or assert the 'disciplines of normality' (Wendell, 1996:87). Wendell also reminds us that experiences of living within a body that suffers should not be ignored in favour of representation. Hall (2011:8) provides some guiding questions for feminist disability studies:

What is the relationship between gender and disability? What role does gender play in the experience of disability? How is gendered disability and dis- or en-abled gender racialized? How do institutions, global economic inequalities, and ideas of citizenship and the nation produce gendered, raced, and classed disability? How does (or should) feminist disability studies address the body's materiality?

Hall (2011) rightly does not intend these questions to be exhaustive. In postcolonial and developing contexts we might, for example, ask questions about inequality which would disrupt monolithic representations of people with disabilities and recognise the impact of settler colonialisms to produce classed and racialised hierarchies of disablement. Intersectional analyses should thus routinely incorporate able-bodiedness with the same urgency as gender, race, sexuality, nationalism or class.

Postcolonial feminist disability studies would be attentive to how disability discourse extends imperialism and revivifies categories of the human that are oppressive and hierarchical (see Parekh, 2007). Sherry (2007) cautions that sophisticated analyses of the connections between postcolonialism and disability are required which go beyond interchangeable conceptions of oppression but should rather interrogate power relations. Sherry (2007) advises that possible questions might be the rhetorical strategies that enable a geography of blame with regard to race and disease, as has been evident in the gendered discourse on HIV/AIDS; racist responses in health care which lead to disability or increase mortality and morbidity; or discourses on immigration that use disability as a marker of fitness for citizenship. Further, disability studies could 
incorporate nuanced postcolonial insights on the fluidity of agency, resistance and power.

We should thus also ask questions that go beyond abjection. Overwhelmingly, and particularly in developing contexts, people with disabilities tend to be represented as abject and suffering subjects. At the other end of the continuum, representations are of 'supercrips' or superheroes who have overcome the deviancy of their bodies to live normal or exceptional lives in spite of their impairments (McDougall, 2006). We rarely read work that details disabled people's strategies to negotiate and be incorporated into their social milieus. A feminist disability studies will also provide alternate representations and stories. Notable examples that re-imagine bodily difference is the work being done by Flatfoot Dance Company (in this issue) and Marlene le Roux and Lucie Pavlovitch's (2008) beautiful coffee table book Look at Me, which showcases the sensuality of women with disabilities, and from which volume our cover photo comes.

Shuttleworth et al (2012) have noted that disability as a category should be disaggregated to ask how particular gendered expectations are manifested by people with particular disabilities. For example, a woman with a physical disability tends to be stereotyped as asexual, whilst a woman with an intellectual or emotional disability is treated as hypersexual. These paradoxical and contradictory responses to disabled femininity draw on notions of embodiment and gendered ideologies of beauty, motherhood and reproduction, representation, discourses on rationality, autonomy and choice, amongst others. This fine attention to difference and human variation will only enhance feminist disability studies.

\section{Localising disabilitystudies}

Although there is a long and proud history of disability activism, disability studies in sub- Saharan Africa is an emerging field. There are numerous challenges in doing disability research in Africa which Leslie Swartz (2014) details as: (1) a lack of experienced researchers; (2) problems with enumerating disability; (3) the overreliance on insider accounts and the problems of representation in small case studies or using individuals' experience on which to base policy; (4) expectations which ignore the challenges of research in resource-poor environments; and (5) the politics of evidence should be grounded in sound methodology.

A Southern African Federation for the Disabled (SAFOD) literature review conducted by Chalklen et al (2009) on disability research in sub-Saharan Africa found that research is uneven across the continent. South Africa was the biggest producer of literature and research, and most disability studies on subSaharan Africa were not produced by African researchers. There was no research 
that focused on women and disabilities, and where women were mentioned it was with regard to sexuality or violence.

However, there have been positive developments in disability studies research in general. The African Network for Evidence to Action on Disability (AfriNEAD) was inaugurated in 2007 (Kachaje et al, 2014). AfriNEAD is an African-based network which seeks to connect research with policy and develop methodologies that transform the lives of people with disabilities. The network brings together a range of stakeholders - academics, researchers, and activists - in a series of triannual symposiums and workshops. An AfriNEAD initiative, the African Journal of Disability, began publishing work from the continent in 2012. AfriNEAD uses the United Nations Convention on the Rights of Persons with Disabilities (UNCRPD) as a frame for its activities. For the 2011 symposium the UNCRPD was compressed into seven themes (see Kathaje et al, 2014) - none of which highlighted gender.

To reiterate, there are very little data on gender and disability within Africa and the developing world, and Emmett and Alant (2006) claim they are mostly anecdotal. Where empirical work has been done a disaggregation by gender is often thought to constitute a gendered analysis, even if a study retains sexist biases, does not mobilise feminist literature or recognise gendered power dynamics. In a just-extract-women approach, gender is often treated as synonymous with women. It may be necessary to develop workshops and toolkits to develop the convergence of these two critical epistemologies.

In South Africa the intersection of gender and disability was represented by two chapters in Melissa Steyn and Mikki van Zyl's 2009 edited volume, The Prize and the Price: Shaping Sexualities in South Africa. In a beautifully written piece Reinette Popplestone (2009) enables a glimpse into her experience of sexuality as a blind woman. Sait et al's (2009) chapter shows how challenging it is for mothers to raise disabled daughters in the impoverished Northern Cape with minimal support.

In one of the first edited volumes focused on disability in South Africa, Disability and Social Change: A South African Agenda, Emmett (2006) looks at the relationship between gender, race, poverty and disability. Although not explicitly read through a feminist lens, Mgwili and Watermeyer's chapter deals with physically disabled women's experiences of discrimination in accessing reproductive health care. Another notable chapter in this volume, although not read with a gendered lens, was an evaluation of the Sexual Assault Victim Empowerment Programme which assists intellectually disabled people with sexual assault cases, by Dickman et al (2006). This programme, run by the Cape Mental Health Society, also provides education on intellectual disability to police officers and legal officials. 
A study by Naidu et al (2005) reports on women with disabilities' experiences of violence and also shows GBV producing disability. One of the few articles on masculinity and disability is Ken Lipenga's (2014) 'Disability and Masculinity in South African Autosomatography' in African Journal of Disability. In Agenda Sue Philpott (1994) described the burden of care by mothers in Amaoti and the implications for community-based rehabilitation programmes. Recently Paul Chappell (2015) explored the connection between queer theory and disability studies for the African context.

Needless to say this is not a comprehensive listing, but merely an attempt to begin to map some of the work that precedes this Special Issue.

\section{Overview of the issue}

Papers in this special issue speak to the complexity and nuances of the intersections of disability with gender, culture, sexualities, poverty, citizenship and other forms of social identity. The studies presented in the articles and/or arguments made, across different geopolitical and material contexts, speak to the salience of the stigmatised representation of disability and how that is specifically gendered, so that women with disabilities are represented differently to men with disabilities and, as illustrated here, experience such disability in different ways. Experiences are, however, also interwoven with material inequalities and other forms of social identity and inequality. A number of themes may be recognised in the body of work presented here, in many cases emerging in more than one paper, and include representation, intersectionality, sexuality, motherhood and resistance.

Talia Meer and Helene Combrinck's article illuminates many of these intersectional aspects of the representation of disabled women, to make a powerful argument for the relationship between negative constructions of gender and disability and women's vulnerability to GBV. The particular focus of this article is on women with intellectual disabilities, and the authors draw on a large study with service providers in the disability and GBV sectors in three provinces of South Africa, to explore reports and perceptions of disabled women's vulnerability to abuse and violence.

The authors argue that the complex combination of the effects of disability and related negative social constructions together with male dominance, sexism and misogyny result in disproportionate exposure to GBV of women with intellectual disability. The article documents many of the myths and perceptions of disabled women that emerge in other articles in this special edition as well, including: the construction of women (and men) with disabilities as less valuable; cultural myths and superstitions about disability; negative constructions of 'disabled' sexuality; questions about the credibility of persons with 
intellectual disability; and women's tendency to internalise the social stigmatising discourses. The authors flag the relationship between intellectual disabilities and continued dominant negative social attitudes and representations as a key concern in prevention of and responses to violence against women with intellectual disabilities.

In the next article Jacqueline Moodley and Laura Graham present a further argument around the intersectionality of gender and disability through a focus on the material contexts of poverty in the South African context. Extracting data from a national survey, the South African National Income Dynamics Study, as part of a larger study on poverty and disability, the authors show how overlapping contexts of poverty and disability shape different outcomes for women than men. Using the indicators of education, employment and income, the study illustrates the intersection of poverty, gender, age, race and disability, foregrounding evidence for more negative outcomes for disabled women, in particular Black women. The study provides empirical support for the argument that gender compounds the effects of disability for Black, poor women.

Also focused on the overlapping representations of gender and disability through a case study of the infamous Oscar Pistorius murder trial, Maretha de Waal deconstructs the gender and disability stereotypes deployed by key role players in the court room. De Waal unpacks the functioning of notions of hegemonic masculinity and disability stereotypes as articulated in arguments by both the prosecution and the defence. She argues that the prosecution based its arguments on a picture of Pistorius that draws on stereotypes linked to his class, age and gender, in which he is presented as 'violent, reckless and sexist'. Similarly, the defence is shown to draw on a narrative that challenges dominant versions of his class, age and masculinity, presented as different to what is expected of dominant masculinity of his class and age.

De Waal argues further how the intersecting discourses on disability that are drawn on by both similarly reproduce disability stereotypes, where Pistorius is portrayed as someone who has undermined the impact of disablism by his sport success, while the defence draws on notions of his vulnerability as a disabled man.

Constructions of sexuality, both discourses that erase sexuality of women with disabilities and those that represent disabled women in hypersexualised terms, are central in the larger framework which privileges able bodies and sexualities and 'others' disabled people, and women in particular, elaborated above. Sexuality emerges as a key theme in a number of the papers in this issue. Drawing on a qualitative study with a group of South African women students with disabilities, Maheshvari Naidu presents an account of a group of young women's narratives on sexuality and disability. Sharing participants' poignant reflections, 
Naidu shows how participants' experiences are complex and layered, representing a co-construction with the able-bodied community. The paper foregrounds the way in which participants negotiate their sexual desires and experiences within the framework of stigmatising discourses of an ableist society which constructs them as asexual or sexually invisible. The stories presented illustrate the way in which participants may in some instances both accept stigmatising discourses about their bodies and sexuality, whilst at the same time resisting and challenging these through more affirming and positive experiences and aspirations. The author argues for what she terms a 'social-sexual model' to provide a more complex perspective on the enmeshment of body, sexuality and disability.

In a similar vein Gbenga Afolayan's article focuses on issues of relationship and sexuality for young women with disabilities in the context of South-Western Nigeria. Based on a large qualitative study with 40 young disabled women, the paper explores contemporary social constructions of disability and gender in this context, and how these unfold in participants' experiences of interpersonal relationships. Analysis of the findings shows how stigmatising representations of women with disabilities as 'helpless, incompetent, asexual and intellectually challenged' continue to have a negative impact on their lives. Similar to papers in other contexts, the author argues that disablist and gender discourses and stereotypes act to rationalise and reproduce hegemonies of male dominance and able-bodiedness.

Continuing with a similar focus, Christine Peta, Judith McKenzie and Harsha Kathard of Develop Africa 2020 apply a feminist intersectional lens to a case study of a disabled woman in Zimbabwe to unpack her experiences of sexuality, relationships, family and work life. The disturbing life story elaborated in detail in this paper shows how disablist discourses are enmeshed with gender norms and stereotypes, class and culture to facilitate a particular vulnerability to violence and un safe sexual practices and challenges in finding secure and loving relationships. Tsitsi's story, as a narrator who is a disabled mother and mother of a disabled child, also raises the way in which pregnant and parenting disabled women may be further stigmatised and abused in healthcare contexts, a theme also evident in Mavuso and Maharaj's paper (see below). On the other hand, the case study also shows how women may resist being victimised and stigmatised and develop areas of strength, such as through entrepreneurial activities, to facilitate agency for themselves and their families.

Sibusisiwe Siphelele Mavuso and Pranitha Maharaj turn to a focus on sexuality in the context of health care, reporting on a qualitative study that focuses on disabled women and men's experiences of sexual and reproductive health services in Durban. The paper reinforces findings internationally that suggest that persons with disabilities are marginalised by sexual and reproductive health programmes. Based on in-depth interviews with a group of sexually active men and women, the 
study documents a gap between participants' needs and the services provided, which impact to a greater degree on women. The authors argue that gender exacerbates the negative experiences, with women participants reporting that health providers respond negatively to their request for family planning services and deny them choices of birth control methods. The study points to a need to address the provision of only equal and supportive access to sexual and reproductive health services for women with disabilities.

This theme of motherhood, emerging in a number of the papers, is taken up by Clare Harvey through a critical literature on subjective experiences of mothers who have disabled children. In her focus she argues for the value of a feminist psychoanalytic lens on mothers of disabled children. The author points out that there is no literature that specifically applies a psychoanalytic lens to the intra- and inter-subjective experiences of mothers raising a child with a disability. She further argues the lack of work across disciplines that explores maternal experiences and subjectivities from the standpoint of the mother.

Harvey provides a comprehensive review of current literature on motherhood experiences, attempting to synthesise the large body of interdisciplinary work on the complexity of motherhood within continued heteronormative societies which make women primarily responsible for care of children. She foregrounds in particular the value of psychoanalytic accounts which theorise the ambivalences of motherhood in general and how that has received minimal attention, given the general social idealisation of motherhood founded on an erasure of any negative or ambivalent feelings by mothers towards their children.

Harvey's paper provides a comprehensive overview of the small body of existing sociopsychological literature on parents (mothers and fathers) of disabled children within the framework of the need for more psychoanalytic literature on such experiences. She concludes by highlighting the importance of challenging deficit approaches and generating more constructive accounts of the way in which parents negotiate parenting a child with a disability in a disablist world, thus calling for further research.

Shanaaz Majiet and Adelene Africa's paper, like many in this issue, raises a wide range of stigma and discrimination experienced by women with disabilities in different contexts. Turning their lens to a particularly neglected area of research, that of a focus on women with disabilities in leadership, they show how a group of women active in organisations in Zimbabwe face multiple challenges to become leaders and sustain their agency and leadership. Yet, as in other studies, participants showed remarkable resilience in the face of ongoing and historical challenges. The study also notably raised the importance of developmental and training resources and support for women with disabilities, particularly within contexts of rural poverty, in assisting their progress and overcoming challenges constraining their access to leadership. 
Importantly a number of the articles point to narratives, experiences and representations of disability, and gendered disability in particular, the serve to destabilise and challenge the negative and 'othering' ones that dominate in and characterise many of the other articles. In a refreshing look at popular representations of women with disability, Ken Lipenga's article on a creative piece of work on the Truth and Reconciliation Commission, the award winning film, Zulu Love Letter (2005), unpacks the way in which the character of Simangaliso, the Deaf character' in the film, serves as "an enabling presence, employing her imaginative abilities to forge links between families, generations and traumatic histories". The author argues that this facilitative role, foregrounded in the film, allows not only the articulation of voices by those silenced by history (such as disabled women) but also serves as resistance to her position as 'other' in her community.

The briefing by Lliane Loots provides a case study that represents a challenge to dominant representations of disabled women through a further creative medium, that of dance. It documents a programme called LeftFeetFIRST which provides a dance educational and performance environment for young disabled dances. The authors presents the case study as an example of a feminist engagement with critical dance studies, which arguably serves to challenge hegemonic assumptions of 'correct' dancing bodies as well as works with gender normative practices like challenging male dominance in dance performance, thus presenting dance as a potential social justice pedagogy foryoung women (and men) with disabilities.

The opinion piece by Gabisile Mkhize serves as a resistant gesture as the author makes the case for problematizing the rhetorical representation of people with disabilities and in particular the way in which the intersection of gender and disability serves to devalue women with disabilities in gendered and ableist global contexts. The author intends to open debate on how the language we use codifies our understanding of disability. Institutions like the media play a vital role in transmitting information, and because of their reach and influence should label and represent people responsibly. Unlike proponents of the social model, who state that the term 'disabled people' critiques disabling contexts, she argues that the label 'disabled people' is reductionist and produces stigma. Instead the author calls for the use of 'individuals with disabilities' so as to signify that disability is not the entirety of an individual's existence, as persons have multiple capabilities and potentialities.

Adhis Chetty's poems encapsulate the range of issues and affective responses that disability engenders. Read together, the poems powerfully sketch the temporariness of able-bodiedness and the force of stigma. In Being White the poet shows how colourism in race discourse has spaces of exception from the vantage of albinism, the dangers of fetishising difference, and that it is society's hostile gaze that is disabling. She explores the thin line between able-bodiedness and disability in Green Stems 
Brown, Brown Stems Green. Bolted Doors makes intriguing connections between GBV and treating women with physical disabilities as undesirable.

\section{Conclusion}

We hope that this collection of papers signals the beginnings of a postcolonial feminist literature on gender and disability in Africa. Undoubtedly we require more gendered analyses of disability and more disabling analyses of gender. There is thus much work ahead to formulate a sophisticated, supple and critical toolkit with which to analyse the intersection of disability with other identities for a postcolonial context. To do so requires developing definitions and models that take the local context seriously.

For example, notions of liberal individualism which underpin much of Northern theorising may not hold the same explanatory power for our contexts. Our societies are not homogenous either, and thus staples of Northern gender and disability literature with regard to the social position of women may not hold true within settler colonial societies like South Africa.

Moodley and Graham's article disrupts the truism that all disabled women are equally disadvantaged and are more so than disabled men, as apartheid racial categories still impact social realities. Black disabled men within South Africa are more likely to experience harsher social conditions and have less social capital than white disabled women. Which brings us to a related point: we need analyses of both femininity and masculinity to explore questions around the making of gendered regimes and differences within multicultural societies. And we should not necessarily focus only on the suffering of people with disabilities - which also reflects a reductionist view of disability - but also show the ingenious ways in which disabled people negotiate their social conditions and create joy and love and dignity and community.

Further, wherever possible we should also disarticulate disabilities and not create homogenous interchangeable subjects. The range of difference opens up multiple avenues for inquiry and activism. Taking the multiplicity of difference seriously also requires a range of ethical responses and responsibilities as we engage with what it means to be human and strive to incorporate multiple others so they may enjoy the fruits of citizenship and recognition.

\section{Note}

1. There are discrepancies in the measurement of differences by sex in the two surveys done: Global Burden of Disease, female prevalence is $11 \%$ higher than males, but the World Report on Disability estimates 60\% more women than men (p. 31). The gap in 
results requires interrogation. The WHO (2011) explanation is that there was a difference in response categories. 